\title{
Is Pain Harder To Withstand Than Tinnitus?
}

Henk M Koning ${ }^{1 *}$

Bas C ter Meulen²

\begin{abstract}
Objectives: The object of the study was to compare the perception of tinnitus with the perception of pain and to find specific factors that could influence the perception of both pain and tinnitus.

Design: A retrospective analysis of 124 patients with tinnitus as main complaint and 300 patients with cervical pain as main complaint who visited our clinic in a two-year period.

Results: This study indicates that it is harder to withstand cervical pain than tinnitus with a higher prevalence of fatigue and impaired work performance in cervical pain patients. Our analyses highlight the importance of fatigue as a potential mediator of the deleterious effects of pain and tinnitus on individual functioning. Female gender and the presence of cervical pain makes patients more accessible for fatigue. Tolerance against tinnitus depends on the perceived maximal intensity of tinnitus, but also of the presence of cervical pain. Cervical pain may reinforce the irritating awareness of tinnitus.

Conclusions: Tinnitus and chronic pain are related to structural and functional brain changes that show a striking overlap between both conditions. The tolerance, the ability to withstand the "unpleasantness" of the percept, for tinnitus and pain depends on the perceived intensity of the sensation. However, the perceived intensity of pain provoked annoyance earlier compared to the perceived intensity of tinnitus. Cervical pain may reinforce and maintain the negative awareness of tinnitus. For a better withstanding of tinnitus, we advise to reduce the perceived maximal intensity of tinnitus and to treat cervical pain and fatigue.
\end{abstract}

Keywords: Tinnitus, cervical pain, tolerance, perception, fatigue, frontostratial circuit. 


\section{INTRODUCTION}

Phantom perception is the awareness of a percept without an external stimulus ${ }^{1-3}$. The vast majority of sensory phantoms are in the somatosensory (phantom pain) and auditory (tinnitus) modalities. Both pain and tinnitus have sensory (discriminative) and affective (the "unpleasantness") dimensions and can induce an avoidance behavior". The perception of pain and tinnitus can be objectivated by the following measures: perceived intensity and tolerance (i.e. the ability to withstand the "unpleasantness" of the percept $)^{3-5}$. The perceived intensity can be measured by the Visual Analog Scale (VAS) and tolerance can be estimated by self-report of the patient ${ }^{6-8}$.

There are meaningful dissimilarities between tinnitus and chronic pain ${ }^{9}$. Pain is a vital protective function and carries an inherently negative feeling ${ }^{10}$. Auditory signals may only become bothersome if the sensation last for extended periods of time ${ }^{9}$. Therefore, the tolerance for pain and for tinnitus may differ. The aim of the study was to compare the perception of tinnitus with that of pain and to find specific factors that could influence the perception of both pain and tinnitus.

\section{MATERIALS AND METHODS}

The Medical Ethics Committees United (Nieuwegein, the Netherlands) agreed this observational retrospective study (W20.131, June 10, 2020).

\section{Subjects}

All patients who came forward to Pain Clinic De Bilt, The Netherlands, for cervical pain or for tinnitus in a two years period (1/1/2018 - 31/12/2019) took part in the study. There were no exclusion criteria. The work-up of patients being a standardized clinical history, the perceived intensity of pain or tinnitus, and a standardized questionnaire for the psychosocial status. The minimal, maximal and mean intensity of tinnitus or pain were measured by the Visual Analogue Scale (VAS). A standardized questionnaire for the psychosocial consequences of tinnitus or pain included the following questions ("Are you good awake in the morning?", "Is your concentration disturbed?", "Is it hard to withstand your tinnitus/pain?", "Are you feeling depressed?", "Are you having fear", and "Are you feeling fatigue?", "Is your work performance impaired?"). Possible answers for each question were none, slight, moderate, or always.

\section{Data Assessment}

Data obtained from these patients are age, sex, the minimal, maximal and mean intensity of tinnitus or pain measured by the VAS, and the results of the psychosocial questionnaire. The VAS of tinnitus or pain are $10-\mathrm{cm}$ lines between at the left end by "no tinnitus or pain" and at the right end by "unbearable tinnitus or pain". The patient indicate with marks the level of the mean, minimal and maximal perceived intensity of his or her tinnitus or pain. The score is measured by the distance (millimetre) on the 10-cm line between the "no tinnitus or pain" anchor and the patient's mark. The questions of the questionnaire for the psychosocial consequences of tinnitus or pain were rated with none (0), slight (1), moderate (2), or good (3). A score of 2 or more indicates for withstanding tinnitus or pain, sleep, concentration, depression, fear, fatigue, and work performance as disturbed.

\section{Statistical Methods}

Statistical analysis was executed with Minitab 18 (Minitab Inc., State College, PA, USA). Student's t-test was employed for continuous variables and $\mathrm{X} 2$ test for dichotomous variables. Discriminant analysis was used in order to evaluate the correlation of the intensity of tinnitus or cervical pain with the feeling that the sensation is hard to withstand and with fatigue. A value of $P$ less than 0.05 was statistically significant.

\section{RESULTS}

In two year time, 124 patients attended our clinic with tinnitus and 300 patients with cervical pain as main complaint. In Table 1, we compared both groups with each other. Cervical pain begins at a younger age and it affects more female (M:F-ratio of 0.5 vs 1.3), compared to tinnitus patients. Although the perceived mean and minimal intensity of cervical pain was less compared to that of tinnitus, it was harder to withstand cervical pain than tinnitus There were more fatigue and more impaired work performance in cervical pain patients compared to tinnitus patients. Multivariate statistical analysis indicated that the perceived maximal intensity of the sensation together with the sensation itself (tinnitus or pain) was statistically significant related to the feeling that the sensation is hard to withstand. Discriminant analysis was used in order to evaluate the correlation of the maximal intensity of the sensation with the prevalence of the feeling that the sensation is hard to withstand for tinnitus and for cervical pain (Figure 1). If the VAS of the intensity of maximal cervical pain is higher than 77 millimetre or if the VAS of the intensity of the maximal tinnitus is higher than 93 millimetres, the prevalence of the feeling that it is hard to withstand the sensation increases from $45 \%$ to $72 \%$.

Patients with cervical pain as leading complaint: Multivariate analysis in patients with cervical pain as principal complaint showed that the VAS of the mean cervical pain and gender were statistically significant for fatigue. In women, a VAS of mean cervical pain exceeding 39 millimetre raises the prevalence of fatigue from $57 \%$ to $90 \%$ and with a VAS of mean cervical pain of more than 47 millimetre the prevalence of hard to withstand cervical pain increases from $53 \%$ to $77 \%$ (Figure 2). In men, the VAS of mean cervical pain was 70 and 64 millimetres respectively for the same increases in prevalence.

Patients with tinnitus as leading complaint: In patients with tinnitus as major complaint, multivariate analysis indicated that the VAS of maximal tinnitus and the 
Table 1: Patients with tinnitus as main complaint were compared with patients with cervical pain as main complaint.

\begin{tabular}{|c|c|c|c|c|c|c|c|c|}
\hline & \multicolumn{3}{|c|}{$\begin{array}{l}\text { Cervical pain as main } \\
\text { complaint }(n=300)\end{array}$} & \multicolumn{3}{|c|}{$\begin{array}{l}\text { Tinnitus as main complaint } \\
\qquad(n=124)\end{array}$} & \multicolumn{2}{|l|}{ P-value } \\
\hline & Prev. & Mean & SEM & Prev. & Mean & SEM & & \\
\hline Age at the start of the complaint (year) & & 41 & 1.4 & & 47 & 1.3 & 0.001 & $S$ \\
\hline Gender (Male): & $32 \%$ & & & $57 \%$ & & & 0.000 & $\mathrm{~S}$ \\
\hline \multicolumn{9}{|l|}{ Perceived intensity of the sensation $(\mathrm{mm})$ : } \\
\hline - Mean & & 57 & 1.9 & & 65 & 2.4 & 0.016 & $S$ \\
\hline - Minimal & & 32 & 1.8 & & 41 & 2.9 & 0.007 & $S$ \\
\hline - Maximal & & 85 & 1.1 & & 82 & 1.9 & 0.09 & \\
\hline - Variation (Maximal - Minimal) & & 56 & 2.0 & & 42 & 2.9 & 0.000 & $S$ \\
\hline Is it hard to resist the sensation? (Yes): & $66 \%$ & & & $50 \%$ & & & 0.009 & $\mathrm{~S}$ \\
\hline Fatigue (Yes) & $77 \%$ & & & $63 \%$ & & & 0.012 & $\mathrm{~S}$ \\
\hline Disturbed sleep (Yes) & $67 \%$ & & & $60 \%$ & & & 0.263 & \\
\hline Impaired work performance (Yes) & $72 \%$ & & & $54 \%$ & & & 0.002 & $S$ \\
\hline Disturbed concentration (Yes) & $54 \%$ & & & $65 \%$ & & & 0.093 & \\
\hline Feeling depressed (Yes) & $44 \%$ & & & $41 \%$ & & & 0.565 & \\
\hline Fear (Yes) & $19 \%$ & & & $28 \%$ & & & 0.061 & \\
\hline
\end{tabular}

Prev.: Prevalence; SEM: Standard Error of the Mean; mm: millimetre; S: Significant.

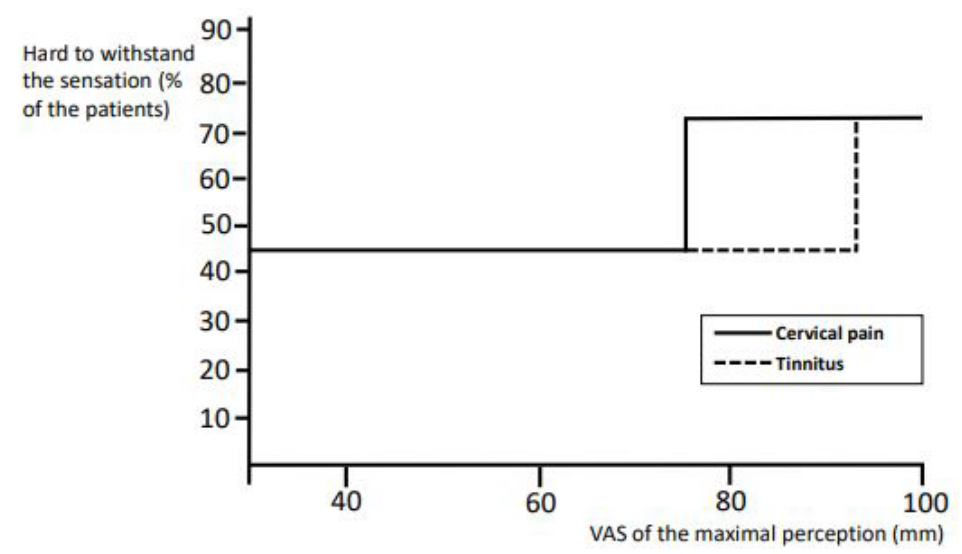

Figure 1: The maximal intensity of the sensation and the prevalence of the feeling that the sensation is hard to withstand for tinnitus and for cervical pain.

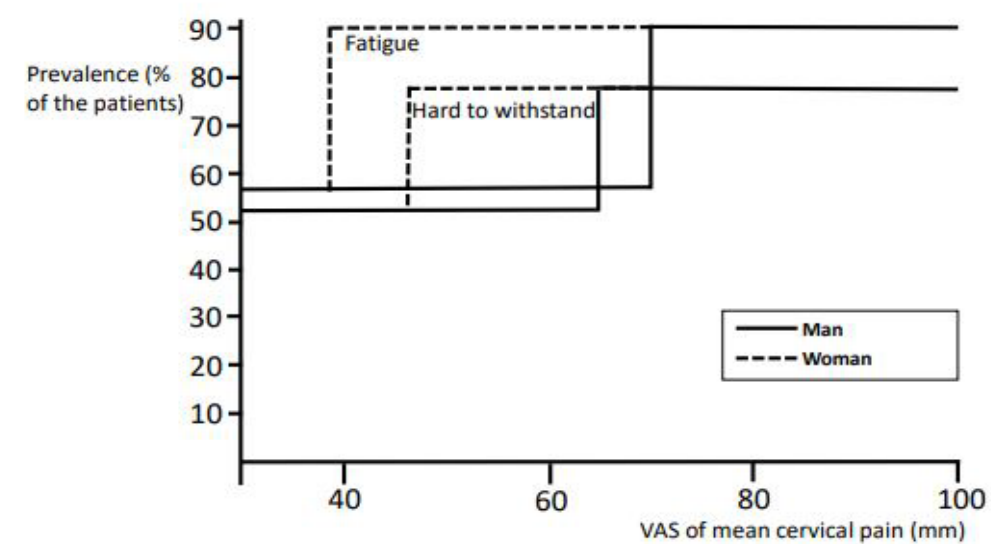

Figure 2: The mean intensity of cervical pain and the prevalence of fatigue and the feeling that cervical pain is hard to withstand for women and men.

presence of cervical pain were statistically significant associated with fatigue. In patients with cervical pain and tinnitus, a VAS of maximal tinnitus exceeding 59 millimetre raises the prevalence of fatigue from $40 \%$ to $88 \%$ and with a VAS of maximal tinnitus of more than 71 millimetre the prevalence of hard to withstand tinnitus increases from $32 \%$ to $65 \%$ (Figure 3). In patients with tinnitus and no cervical pain, the prevalence of fatigue and of hard to withstand tinnitus remained $40 \%$ and $32 \%$, respectively. 


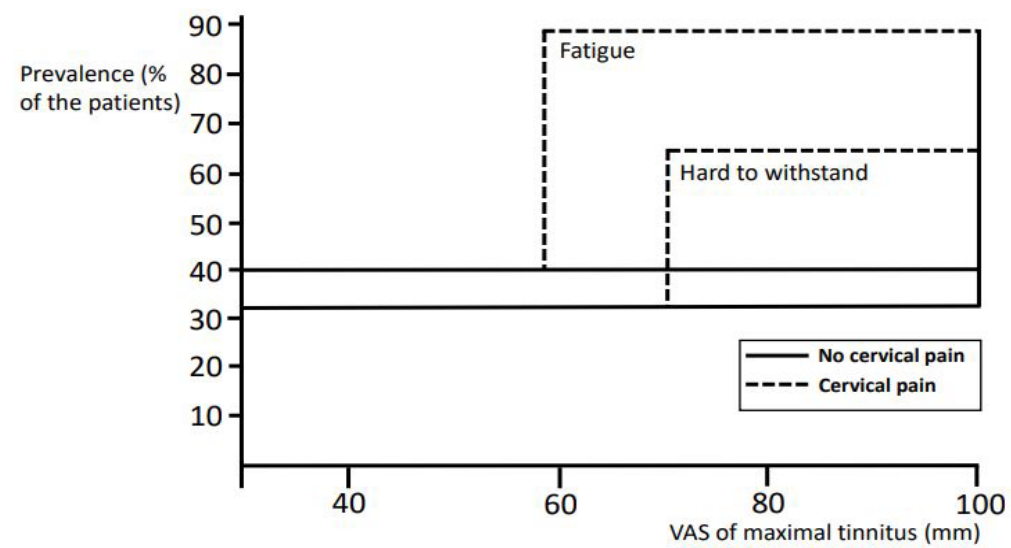

Figure 3: The maximal intensity of tinnitus and the prevalence of fatigue and the feeling that tinnitus is hard to withstand in patients with and without cervical pain.

\section{DISCUSSION}

This study indicates that it is harder to withstand cervical pain than tinnitus with a higher prevalence of fatigue and impaired work performance in cervical pain patients. The feeling that the sensation is hard to withstand is closely related to fatigue. Our analyses highlight the importance of fatigue as a potential mediator of the deleterious effects of pain and tinnitus on individual functioning. Female gender and the presence of cervical pain makes patients more accessible for fatigue. Tolerance against tinnitus depends on the perceived maximal intensity of tinnitus, but also of the presence of cervical pain. Cervical pain may reinforce the irritating awareness of tinnitus.

Tinnitus and pain are sensory-perceptual conditions linked with negative affections ${ }^{9}$. Percepts reach only awareness when increased neuronal activity in the primary sensory or auditory cortex is connected to brain networks ${ }^{1}$. The distress network of tinnitus and pain overlaps each other. A pain or an auditory stimulus delivered near threshold becomes only consciously perceived when the dorsal anterior cingulate cortex and anterior insula are activated. The intensity of perceived pain and auditory stimuli relates to the degree of activity in the dorsal anterior cingulate cortex and anterior insula ${ }^{2}$.

The frontostratial circuits has a pivotal role in the forming and continuation of tinnitus and chronic pain ${ }^{9}$. Especially, the ventromedial prefrontal cortex and the nucleus accumbens estimate the relevance of sensory stimuli and manage the information flow via descending pathways $s^{3-9}$. However, there are also meaningful differences between tinnitus and chronic pain ${ }^{9}$. Pain induces a negative affection for the protection of vital functions. Auditory signals may only become inconvenient when the awareness persists for a long time. Our analysis indicates that the perceived intensity of pain provoked annoyance earlier compared to the perceived intensity of tinnitus.

Male patients could withstand the intensity of cervical pain better than female, especially in the range of VAS of mean cervical pain from 47 to 64 millimetre. Women are at higher risk of both developing and reporting pain ${ }^{11}$. Increased functional connectivity of the prefrontal cortex to the nucleus accumbens is predictive of pain persistence and there are sex-dependent differences in processes that regulate the intrinsic excitability of these neurons ${ }^{9-12}$. Sex differences involve the organization of the insula and anterior cingulate cortex together with the emotional-arousal reactivity in patients with chronic pain ${ }^{13}$. We did not find an influence of gender on withstanding tinnitus.

Tinnitus can be switch off by feedback from the limbic system, which can obstruct the tinnitus signal before reaching the auditory cortex ${ }^{9}$. If the limbic regions are affected, this "noise-cancellation" mechanism can pull down and chronic tinnitus results. Such a limbic gating system also exists for the somatosensory system ${ }^{9}$. It is not only responsible for the unpleasantness of pain but also for modulating its intensity. The ventromedial prefrontal cortex and the nucleus accumbens are implicated in the persistence of pain ${ }^{12}$. Both pain and tinnitus can have an additive effect on the frontostriatal circuits. In our study, the presence of cervical pain made it harder to withstand tinnitus in the range of VAS of mean intensity of tinnitus exceeding 71 millimetres. Tolerance against tinnitus depends on the perceived maximal intensity of tinnitus, but also of the presence of cervical pain.

Our analyses highlight the importance of fatigue as potential mediator of the deleterious effects of pain and tinnitus on individual functioning. A strong positive correlation between fatigue and the perceived intensity of pain and tinnitus was observed. Fatigue is not only associated with the experience of pain and tinnitus, but it may also predict how well people maintain activity in the face of pain or tinnitus ${ }^{14,15}$. Sleep disturbances, resulting in fatigue, strongly predicts lower tinnitus tolerance ${ }^{16}$. Tinnitus may interfere with sleep by the amplification of internally perceived noise during times of low environmental noise or due to an increased focus on tinnitus and undue anxiety ${ }^{16}$. Sleep impairment is also a predictor of longstanding pain, possibly as a result of changes in endogenous pain inhibition associated with an attenuated activation of the nucleus accumbens ${ }^{17}$. 
This study has limitations. The first limitation is its retrospective kind and a prospective follow-up study is advocated to confirm these results and the conclusions. Another limitation is that the lack of measurement of the perceived intensity of cervical pain in patients with tinnitus as major complaint. $A$ VAS-measurement of cervical pain together with a VASmeasurement of cervical pain in this group of patients could better differentiate which influence on withstanding tinnitus is the most. Hereafter, prospective studies are required with an estimate of the intensity of cervical pain in patients with tinnitus.

\section{CONCLUSION}

Tinnitus and chronic pain are related to structural and functional brain changes that show a striking overlap between both conditions. A limbic gating system exists for both the somatosensory system and the auditory system, which is responsible not only for creating the unpleasantness of pain and tinnitus but also for modulating its intensity. The tolerance, the ability to withstand the "unpleasantness" of the percept, for tinnitus and pain depends on the perceived intensity of the sensation. However, the perceived intensity of pain provoked annoyance earlier compared to the perceived intensity of tinnitus. Cervical pain may reinforce and maintain the negative awareness of tinnitus. For a better withstanding of tinnitus, we advise to reduce the perceived maximal intensity of tinnitus and to treat cervical pain and fatigue.

\section{CONFLICT OF INTEREST}

The authors declare no potential conflict of interest

\section{REFERENCES}

1. De Ridder D, Elgoyhen AB, Romo R, Langguth B. Phantom percepts: tinnitus and pain as persisting aversive memory networks. Proc Natl Acad Sci USA. 2011;108:8075-80.

2. De Ridder D, Vanneste $S$, Congedo $M$. The distressed brain: a group blind source separation analysis on tinnitus. PLoS One. 2011;6:e24273.

3. Haider HF, Bojic T, Ribeiro SF, Paço J, Hall DA, Szczepek AJ. Pathophysiology of Subjective Tinnitus: Triggers and Maintenance. Front Neurosci. 2018;12:866.

4. Chayadi E, McConnell BL. Gaining insights on the influence of attention, anxiety, and anticipation on pain perception. $J$ Pain Res. 2019;12:851-64.
5. Dauman N, Erlandsson SI, Albarracin D, Dauman R. Exploring Tinnitus-Induced Disablement by Persistent Frustration in Aging Individuals: A Grounded Theory Study. Front Aging Neurosci. 2017;9:272-4.

6. Raj Koziak D, Gos E, Swierniak W, Rajchel JJ, Karpiesz L, Niedzialek I, et al. Visual Analogue Scales as a tool for Initial Assessment of Tinnitus Severity: Psychometric Evaluation in a Clinical Population. Audiol Neurootol. 2018;23:229-37.

7. Kersten $P$, White PJ, Tennant $A$. Is the pain visual analogue scale linear and responsive to change? An exploration using Rasch analysis. PLoS One. 2014;9:e99485.

8. Darbari DS, Brandow AM. Pain-measurement tools in sickle cell disease: where are we now?. Hematology Am Soc Hematol Educ Program. 2017;1:534-41.

9. Rauschecker JP, May ES, Maudoux A, Ploner M. Frontostriatal Gating of Tinnitus and Chronic Pain. Trends Cogn Sci. 2015;19:567-78.

10. Heitmann H, May ES, Tiemann L, Schmidt P, Nickel MM, Dinh ST, et al. Motor Responses to Noxious Stimuli Shape Pain Perception in Chronic Pain Patients. eNeuro. 2018;5(5):ENEURO.0290-18.

11. Häggman-Henrikson $B$, Liv $P$, Ilgunas $A$, Corine $M$, Durham $F$, Anna $L$, et al. Increasing gender differences in the prevalence and chronification of orofacial pain in the population. Pain. 2020;161:1768-75.

12. Baliki MN, Apkarian AV. Nociception, Pain, Negative Moods, and Behavior Selection. Neuron. 2015;87:474-91.

13. Gupta A, Mayer EA, Fling C, Labus JS, Nalliboff BD, Hong $J Y$, et al. Sex-based differences in brain alterations across chronic pain conditions. J Neurosci Res. 2017;95:604-16.

14. Boggero IA, Rojas-Ramirez MV, Carlson CR. All Fatigue is Not Created Equal: The Association of Fatigue and Its Subtypes on Pain Interference in Orofacial Pain. Clin J Pain. 2017;33:231-7.

15. Lasisi AO, Gureje O. Prevalence of insomnia and impact on quality of life among community elderly subjects with tinnitus. Ann Otol Rhinol Laryngol. 2011;120:226-30.

16. Liu YF, Hu J, Streelman M, Guthrie OW. The epworth sleepiness scale in the assessment of sleep disturbance in veterans with tinnitus. Int J Otolaryngol. 2015;42:94-69.

17. Seminowicz DA, Remeniuk B, Krimmel SR, Smith MT, Barrett FS, Wulff $A B$, et al. Pain-related nucleus accumbens function: modulation by reward and sleep disruption. Pain. 2019;160:1196-207. 